Acta Horticulturae et Regiotecturae 1

Nitra, Slovaca Universitas Agriculturae Nitriae, 2019, pp. 29-36

\title{
SMALL SACRAL ARCHITECTURE AND ITS GREENERY IN LOWER SPIŠ REGION IN SLOVAKIA
}

\author{
Denisa HALA JOVÁ, ${ }^{*}$ Attila TÓTH, Peter HALAJ \\ Slovak University of Agriculture in Nitra, Slovakia
}

\begin{abstract}
Small sacral structures are significant elements of Spiš cultural landscapes (North-East Slovakia). This paper presents results of the field mapping of small roadside sacral objects and their greenery along a 66-kilometre long road section connecting North and South of Lower Spiš and the municipalities of Levoča, Spišská Nová Ves, Hnilčík, Mníšek nad Hnilcom, Smolník, and Úhorná. This road was an old trade route and has an important historical legacy in Lower Spiš - a traditional mining cultural landscape with a characteristic hilly topography, wide forest landscapes and rich mining history. The presented mapping was conducted within the research project VEGA 1/0371/18 "SacralArch: Preservation of the Historical Legacy and Architectural Diversity of Small Sacral Structures in Cultural Landscapes of Slovakia", based on the methodology elaborated by Tóth (2018), which includes localisation, spatial context, technical or health condition and an overall description of the sacral element and the woody plants in its direct surroundings. In total, 13 small roadside sacral objects were mapped, while the most prevailing elements are crosses. The most common cross type is wooden cross without pedestal. The oldest and aesthetically most valuable elements are metal crosses on stone pedestals, made of travertine. The most valuable element is a registered cultural monument - a Baroque roadside chapel of St. John of Nepomuk from 1726 in Smolník. Woody plants accompany only roadside crosses in the study area. The oldest and most significant in terms of landscape value are individuals of small-leaved linden trees (Tilia cordata Mill).
\end{abstract}

Keywords: cultural landscape, heritage, monument, small sacral structure, tree

Small sacral objects arose emerged in our landscapes as symbols of Christian culture and they are abundant especially in traditional Catholic regions (Kyselka, 2014). A high number of small sacral objects in cultural landscapes of Central Spiš can be seen as an evidence of the spiritual richness, belief and religiousness of the inhabitants. Small sacral objects are located in built-up areas or open landscapes (Tóth and Verešová, 2018), they are very abundant in cemeteries (Halajová and Kubišta, 2015) and occur also within or around historical estates and their landscape gardens (Tóth and Feriancová, 2016). Small sacral objects are defined by Matáková (2011) and Dohnálová et al. (2015) as specific architectural objects (e.g. chapels, wayside shrines, little bell towers, architectural and figural compositions), including simpler forms (e.g. crosses, statues of saints, and pictures on trees). The main functions of these objects are:

1. cult (religious) - as symbols of Christian belief;

2. memorial-as commemoration of historic events or persons;

3. votive - as gratitude for God's answer to prayers;

4. warning-conciliation crosses;

5. protection - located on safe sites at crossroads;

6. representation - manifesting the social status of the donor;

7. administrative - marking field boundaries or property borders (Matáková, 2011).
Sacral objects are linked to theplaces where they are located, while creating its their identitiesy. They were not randomly placed, quite the opposite, they were located on important ritual sites that where linked to the life of local inhabitants, on places with good visibility, accessibility, as well as symbolic significance (Kopeček, 2015). In the landscape of Spiš, especially Gothic and Baroque urban structures and architectural objects prevail (Tomaško, 2000). These were strongly influenced by German culture and architecture. Pilgrimage as a phenomenon developed especially in the period of Baroque. Pilgrim routes that led to important pilgrim sites were normally accompanied by small sacral objects, which functioned as stops reminding the meaning and spirit of the journey. Spišs, especially Central Spiš landscape around Spiš Castle is an outstanding example of designed Baroque sacral landscape in Slovakia (Tomaško, 2000; Jančura, 2011; Supuka and Štefunková, 2014). At the vicinity of Spišská Kapitula (cathedral and chapterhouse), a symbolic landscape with seven Passion stops was created in the $17^{\text {th }}$ century. This "Spiš Jerusalem" is the only recorded implementation of Calvario Jerusalem in Slovakia. The spatial setting of these objects copies the Jerusalem Calvary and its elements in an almost identical scale (Jančura, 2011; Jančura and Bohálová, 2001). The cultural environment of Spiš is characterised by the cult of Virgin Mary. The Marian Hill in Levoča, with the gracious statue of the Virgin Mary is a famous pilgrim site of national importance. Small sacral objects and designed cultural landscapes of Central Spiš

Contact address: Denisa Halajová, Slovak University of Agriculture in Nitra, Faculty of Horticulture and Landscape Engineering, Department of Landscape Architecture, Tulipánová 7, 94976 Nitra, Slovakia, e-mail: denisa.halajova@uniag.sk 
are important features of the UNESCO cultural heritage site "Spiš Castle and the associated cultural monuments" that were assessed among others by Tomaško (2000), Kraková (2004), Felberová (2010), and Jančura (2011).

Woody plants are naturally linked to small sacral structures. Trees were significant sacred symbols in pagan cults, as well as in Christian religion. Especially linden trees were planted to protect sacral buildings, for which there is extensive evidence in Bohemia, Moravia and Slovakia. The number of trees is related to the numeral symbolism one as the unity of God - the creator; two as contrast and bipolarity; three/triangle as trinity of God (omne trium perfectum); and four as symbol of the world and universe (Biedermann, 1992).

\section{Material and methods}

The Spiš cultural region is located in North-Eastern Slovakia. In the past, Szepes (Hungarian) / Zips (German) / Spiš (Slovak) / Scepusium (Latin) was an autonomous Royal County of the Hungarian Kingdom and later Austro-Hungarian Monarchy. Its cultural and historic centre is the Spiš Castle. This region is usually divided into three main parts - Lower Spiš (Spišská Nová Ves and Gelnica districts), Central Spiš (Levoča and Poprad districts and the main part of Kežmarok district) and Upper Spiš (Stará L’ubovňa district and the North-Western part of the Kežmarok district - Zamagurie). There are two UNESCO cultural heritage sites:

1. Spiš Castle and the associated cultural monuments (municipalities of Spišské Podhradie, Spišská Kapitula and Žehra);

2. the historic town Levoča.

The area of Central Spiš has already been studied for its cultural monuments and landscape heritage. Therefore, we decided to focus on Lower Spiš with an important mining heritage, hilly topography and a high share of forest land use, which makes it different from the designed landscapes of Central Spiš around Spiš Castle with a prevailing agricultural land use. This paper presents the outcomes of the mapping of small sacral architecture objects along B-road II/533 from Levoča to Gemerská Poloma, on the section Levoča Spišská Nová Ves - Hnilčík; and B-road II/549 from Mníšek nad Hnilcom to Krásnohorské Podhradie on the section from Mníšek nad Hnilcom to Úhorná. These roads pass through the whole area of Lower Spiš, from North to East, and the whole section is $66 \mathrm{~km}$ long (see Figure 1), thus it can be considered a representative research sample of Lower Spiš.

The landscape between Levoča and Spišská Nová Ves consists of agricultural areas (Hornád River basin), hills and forests (Volovské vrchythe Volovec Mountains / Slovenské rudohoriethe Slovak Ore Mountains), deep brook valleys (Železný potok, Hnilecký potok, Smolnícky potok), and a water reservoir (Úhorná). In the hilly forest landscape of Volovské vrchythe Volovec Mountains, there was an important purple-copper-ore mining area with mining settlements that flourished mainly in the $14^{\text {th }}$ and first half of the $15^{\text {th }}$ century. The studied road used to be an important trade route for transporting copper from Spiš to Poland in the $12^{\text {th }}$ century. An old settlement Mníšek nad Hnilcom was named after the monks (mních / mníšek) who lived in Jasov and had hermitages in the area (Vencko, 2018) and who co-formed the sacral character of the Spiš landscape.

The field mapping presented in this paper is one of the main objectives of the research project VEGA 1/0371/18 "SacralArch: Preservation of the Historical Legacy and Architectural Diversity of Small Sacral Structures in Cultural Landscapes of Slovakia". The field mapping methodology was elaborated in a unified way for the whole research project and for all studied areas by Tóth (2018) and consists of the localisation, spatial context and description of the sacral element and woody plants around it, their photographic documentation, and searching for evidence in historical maps.

The overall technical condition of the object and the degree of damage is classified as follows: 0 - good technical condition/no damage - $0 \% ; 1$ - negligible damage - 1-10\%; 2 - low damage - 11-25\%; 3 - medium damage - 26-60\%; 4 - heavy damage - 60-69\%; 5 - considerably damaged or destroyed - 70-100\%.

Trees and shrubs were assessed individually. We focused on their number, species and spatial composition. We measured the trunk circumference $[\mathrm{m}]$ at the height of $1.3 \mathrm{~m}$, the width $(\mathrm{m})$ and height $[\mathrm{m}]$ of the woody plant. We evaluated the age phase ( 1 - young; 2 - outgrown; 3 - adolescent; 4 - mature; 5 - old woody plants) (Šimek,

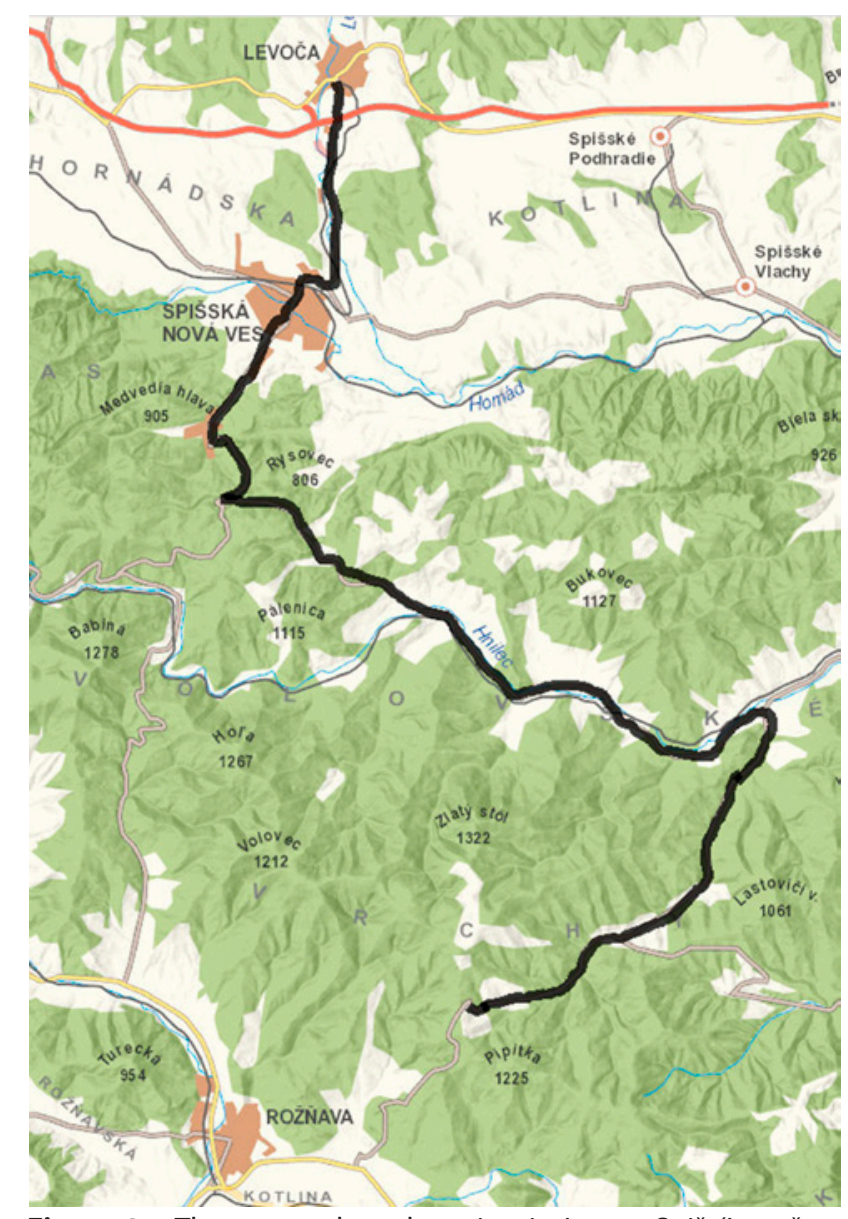

Figure 1 The mapped road section in Lower Spiš (Levoča Spišská Nová Ves - Hnilčík - Mníšek nad Hnilcom Smolník - Úhorná) as research sampling area Source: https://zbgis.skgeodesy.sk 
2001); the landscape value (1 - low; 2 - below average; 3 average; 4 - above average; 5 - extraordinary) (Machovec, 1987); and the overall health condition and degree of damage $(0$ - healthy, $0 \%$ damaged; 1 - negligible damage up to $10 \% ; 2$ - low damage up to $25 \% ; 3$ - medium damage up to $60 \% ; 4$ - heavy damage up to $69 \%$ and 5 - excessive damage $70 \%$ to total damage 100\%) (Juhásová, 2009 amended according to Annex No. 35, Decree No. 24/2003 of the Act No. 543/2002 on Nature and Landscape Protection). For shrubs, we documented the number, species, height and composition. Herbaceous plants were documented by text descriptions. The historical evidence in maps was documented based on the $1^{\text {st }}\left(18^{\text {th }}\right.$ century - 1782-1785), $2^{\text {nd }}\left(19^{\text {th }}\right.$ century - 1819-1869) and $3^{\text {rd }}(1869-1887)$ Military Survey of the Austro-Hungarian Monarchy (Tóth, 2018).

\section{Results and discussion}

Along the $66 \mathrm{~km}$ long road section from Levoča to Úhorná, we mapped 13 roadside small sacral objects. These are located in two districts and 7 municipalities - Spišská Nová Ves District/Harichovce (1), Spišská Nová Ves (1), Hnilčík (4); and Gelnica District/Nálepkovo (2), Švedlár (1), Smolník (3), Úhorná (1). Eight of these objects are located in built-up areas, five in the open landscape (see Table 1).

The prevailing typology is represented by crosses (11). Two of them are located on crossroads. There are two roadside chapels with niches, located in important settlements - Spišská Nová Ves and Smolník. The most common cross type in terms of material is wooden cross without pedestal, with round tin roof (6) and grey-paintedmetal-cast (4) or polychrome-tin (2) Corpus Christi. Eight crosses are surrounded by a simple wooden fence. The oldest and aesthetically most valuable are two metal crosses on stone pedestals and one stone cross, all located in Hnilčík and made of travertine, in combination with cast-metal Corpus Christi. There are some similarities between the studied area and the area of Central Spiš, mainly in terms of object typology and material, while in Lower Spiš common crosses prevail. This can be explained by the fact that there are not such historically significant towns and cities as in Central Spiš, where Kraková (2004) identified 67 small sacral objects in 12 municipalities around the Spiš Castle - common crosses (23), memorial crosses (11), chapels with niches (13), roadside shrines (12) and chapels with an inner space (8). Here, the number of chapels is significantly influenced by the Spiš Jerusalem. Similarly to Lower Spiš, the memorial crosses are mostly made of travertine. Common crosses are made of wood or metal, with a tin roof, a cast-metal or tin Corpus Christi and they are normally surrounded by a small ornamental garden.

It is important to highlight travertine as a regional specificity of Spiš, which was extracted here in the past for sculpture and stonemasonry purposes. This had an impact also on the material character of many small sacral objects all over Spiš. According to Felberová (2010), travertine is the most common material of stone crosses in Spiš, while sandstone crosses occur mainly around Levoča and Spišské Podhradie.

The oldest and aesthetically most valuable cross (No. 4) in the study area is located on the crossroad of II/533 (Levoča Gemerská Poloma) and II/546 (Hnilčík - Prešov), at the edge of a spruce forest (see Figure 2). This cross dates back to 1907 and it was obviously raised on the site of a previous cross, which is documented in the $3^{\text {rd }}$ military survey (1869-1887).
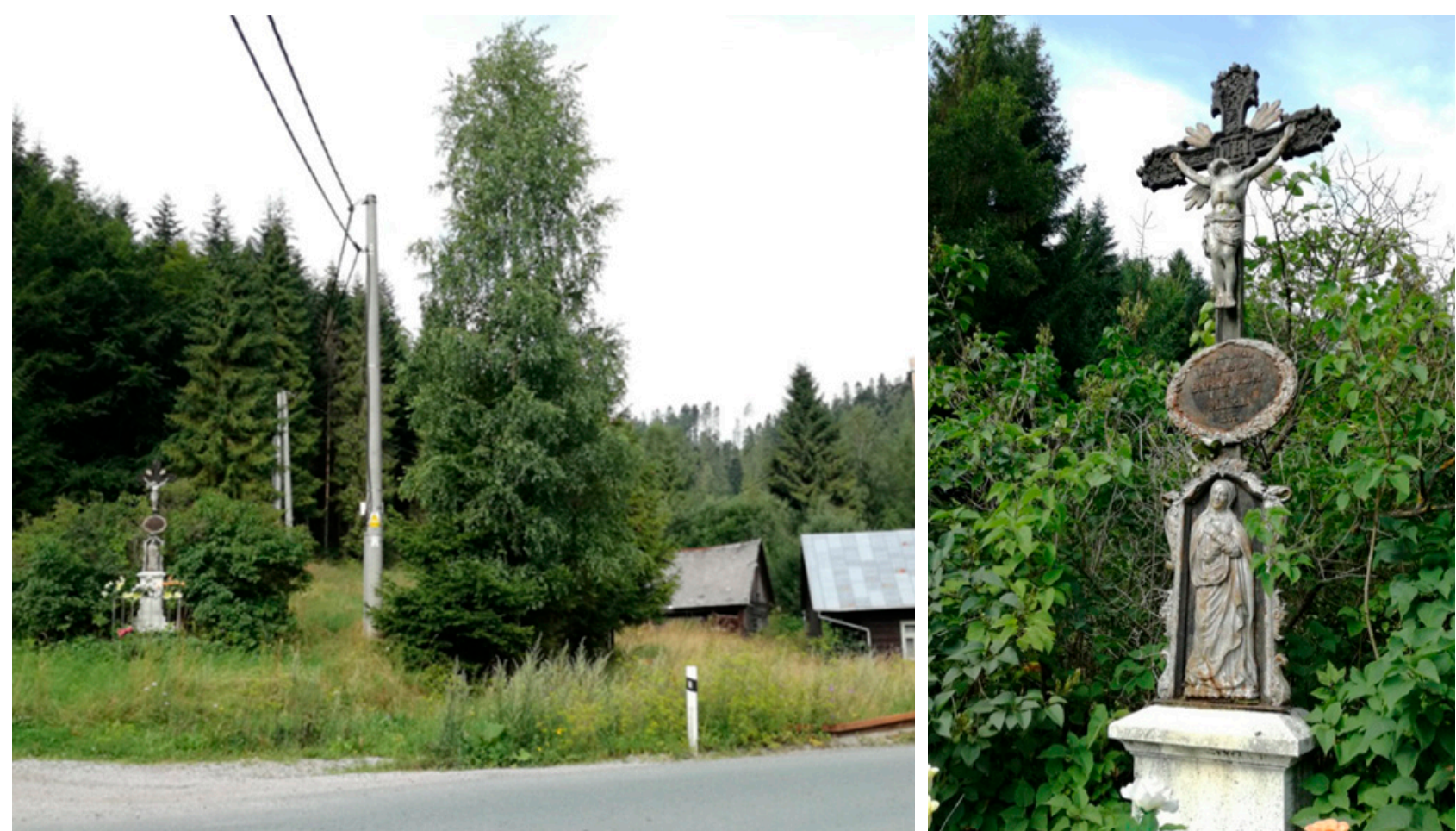

Figure 2 Blac-painted metal-cast cross (No. 4) on a white stone pedestal made of travertine located on the crossroad of B-road II/533 (Levoča - Gemerská Poloma) and B-road II/546 (Hnilčík - Margecany) Photo: Halajová, 2018 


\begin{tabular}{|c|c|c|c|c|c|c|c|c|c|c|c|c|c|c|}
\hline \multirow{3}{*}{ 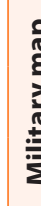 } & $\angle 88 \mathrm{~L}-698 \mathrm{~L}_{\mathrm{p},} \varepsilon$ & $\stackrel{\check{\Sigma}}{\check{\nu}}$ & $\stackrel{\check{\nu}}{\stackrel{u}{\Sigma}}$ & $\stackrel{u}{\Sigma}$ & $\stackrel{u}{\Sigma}$ & $\stackrel{\check{\Sigma}}{\check{\Sigma}}$ & 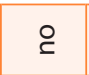 & $\stackrel{\circ}{\check{2}}$ & $\stackrel{\check{\Sigma}}{\beth}$ & 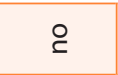 & $\stackrel{u}{\Sigma}$ & $\stackrel{\check{x}}{x}$ & $\stackrel{\check{\nu}}{\supset}$ & 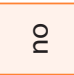 \\
\hline & $698 \mathrm{~L}-6 \mathrm{LI} \mathrm{L}_{\mathrm{pu}} \mathrm{Z}$ & $\stackrel{\circ}{\complement}$ & $\stackrel{\check{\nu}}{\beth}$ & $\stackrel{\circ}{\check{2}}$ & $\stackrel{\circ}{\check{1}}$ & $\stackrel{\circ}{\check{1}}$ & $\stackrel{\circ}{\check{2}}$ & $\stackrel{\circ}{\check{c}}$ & $\stackrel{\circ}{\check{1}}$ & $\stackrel{\circ}{\check{2}}$ & $\stackrel{\circ}{\check{1}}$ & $\stackrel{\circ}{\check{2}}$ & 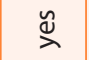 & $\stackrel{\circ}{\complement}$ \\
\hline & s8LL-Z8LL ${ }_{35} L$ & ๖ & 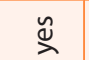 & & 요 & & 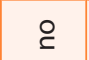 & $\stackrel{\circ}{\complement}$ & 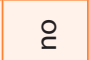 & 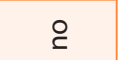 & 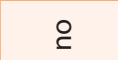 & 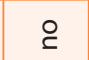 & $\stackrel{\oiiint}{\lambda}$ & $\stackrel{\circ}{\complement}$ \\
\hline \multirow{9}{*}{ 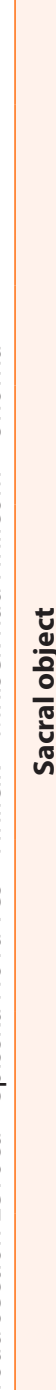 } & 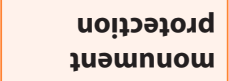 & 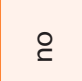 & 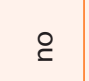 & 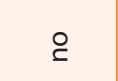 & 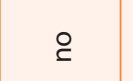 & 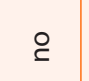 & 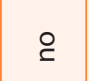 & 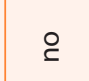 & 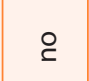 & 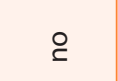 & $\stackrel{\circlearrowright}{\rightleftharpoons}$ & 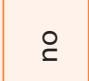 & 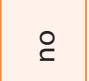 & $\stackrel{\circ}{\complement}$ \\
\hline & би!วиәу & 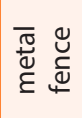 & 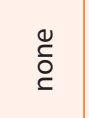 & \begin{tabular}{l}
\multicolumn{1}{c}{} \\
$\stackrel{0}{\check{D}}$
\end{tabular} & 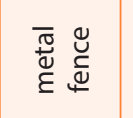 & $\begin{array}{l}\stackrel{\mathscr{c}}{0} \\
\stackrel{5}{\check{c}}\end{array}$ & 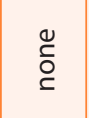 & 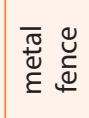 & 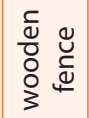 & 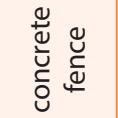 & 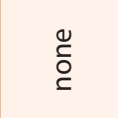 & 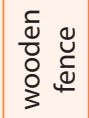 & 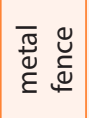 & 龸 \\
\hline & әбешер & 0 & 0 & - & - & 0 & 0 & 0 & 0 & 0 & 0 & 0 & 0 & $\sim$ \\
\hline & 6u!tep & $\stackrel{\pi}{a}$ & $\stackrel{\pi}{s}$ & 옹 & $\stackrel{\circ}{\circ}$ & $\stackrel{\pi}{\Omega}$ & $\stackrel{\pi}{s}$ & $\stackrel{\pi}{\check{c}}$ & $\stackrel{\pi}{\check{c}}$ & $\stackrel{\pi}{\varrho}$ & $\stackrel{\mathscr{N}}{\stackrel{N}{L}}$ & $\stackrel{\pi}{\Omega}$ & $\stackrel{\pi}{s}$ & $\stackrel{\pi}{s}$ \\
\hline & чтр!м/зчб!әч & $\bar{\sigma}$ & 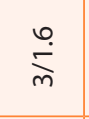 & $\stackrel{n}{\sim}$ & & ถ⿱丶万仒 & 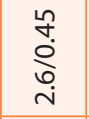 & $\bar{\gamma}$ & $\bar{\gamma}$ & $\bar{m}$ & $\stackrel{n}{\sigma}$ & 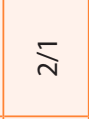 & $\bar{m}$ & $\lesssim$ \\
\hline & 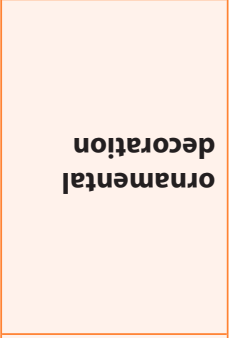 & 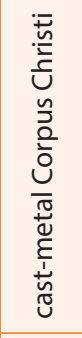 & 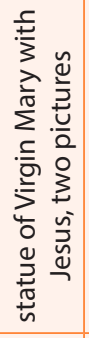 & 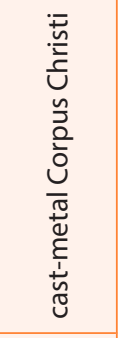 & 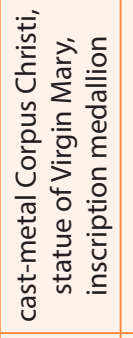 & 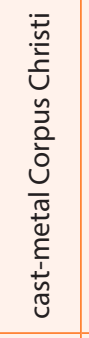 & 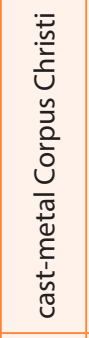 & 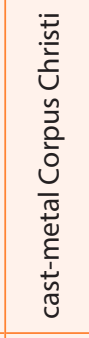 & 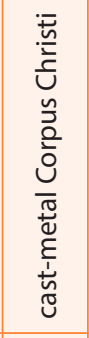 & 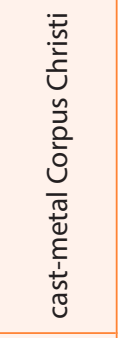 & 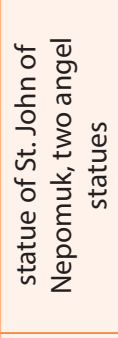 & 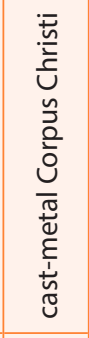 & 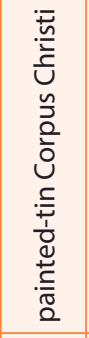 & 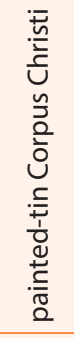 \\
\hline & yṣuy әJеرans & 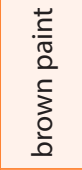 & 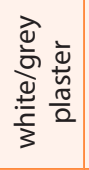 & 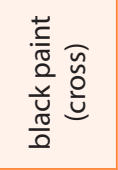 & 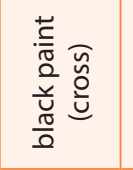 & 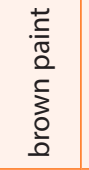 & $\begin{array}{l}\stackrel{0}{\check{c}} \\
\stackrel{5}{\check{c}}\end{array}$ & 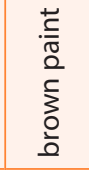 & 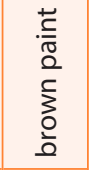 & 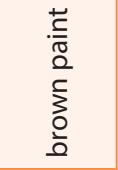 & 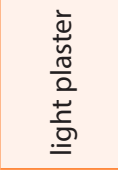 & 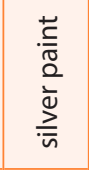 & 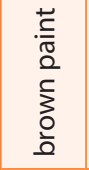 & $\begin{array}{l}\stackrel{+}{\frac{7}{\pi}} \\
\frac{0}{0} \\
\frac{1}{3} \\
0 \\
\frac{0}{0}\end{array}$ \\
\hline & |ердәреш & 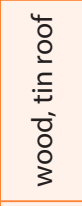 & 訔 & 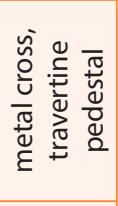 & 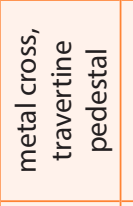 & 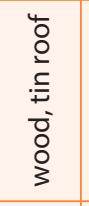 & 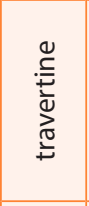 & 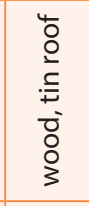 & 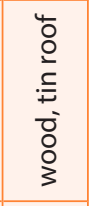 & 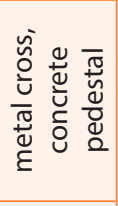 & 訔 & 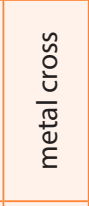 & 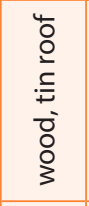 & 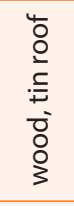 \\
\hline & 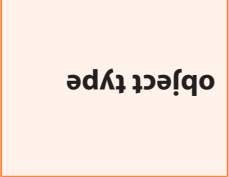 & 气ั̆ & 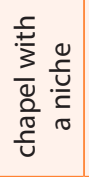 & $\stackrel{\tilde{u}}{\stackrel{u}{u}}$ & $\stackrel{\check{\varrho}}{\underline{U}}$ & 气ू̆ & ôّ & 气ั̆ & $\stackrel{\widetilde{o ̆ ~}}{\underline{v}}$ & $\stackrel{\widetilde{o ̆ ~}}{\underline{U}}$ & 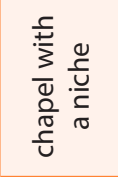 & 气ू̆ & 气ัٌ & $\stackrel{\tilde{\varrho}}{\mathscr{U}}$ \\
\hline \multirow{5}{*}{ ل } & ұхәłuos |e!̣eds & 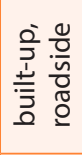 & 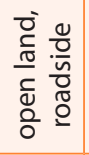 & 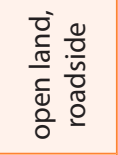 & 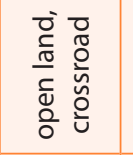 & 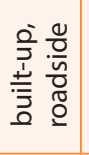 & 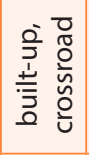 & 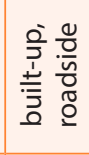 & 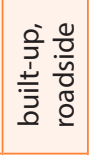 & 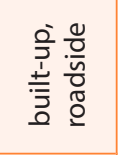 & 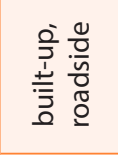 & 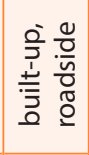 & 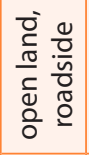 & 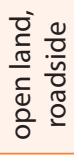 \\
\hline & I·s·e·w & 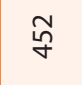 & 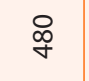 & $\underset{\infty}{\sim}$ & $\frac{0}{\infty}$ & $\hat{\imath}$ & $\stackrel{\infty}{\circ}$ & in & $\frac{\infty}{i n}$ & $\stackrel{8}{f}$ & $\stackrel{\tilde{n}_{n}}{n}$ & 总 & $\frac{8}{6}$ & กู \\
\hline & Sdפ & 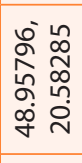 & 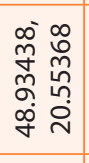 & 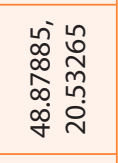 & 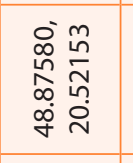 & 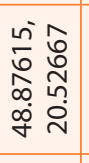 & 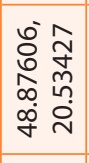 & 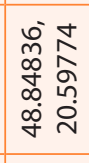 & 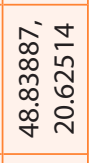 & 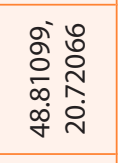 & 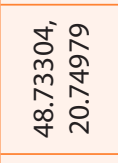 & 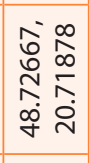 & 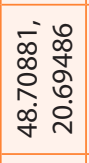 & 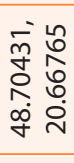 \\
\hline & peod & 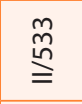 & 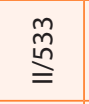 & 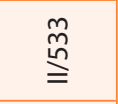 & 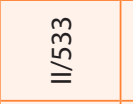 & 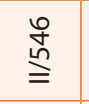 & $\stackrel{\text { ㅇํ }}{\stackrel{1}{=}}$ & $\begin{array}{l}\stackrel{0}{10} \\
\stackrel{1}{=}\end{array}$ & $\stackrel{\text { 苫 }}{=}$ & $\stackrel{\text { 是 }}{=}$ & 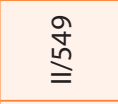 & $\stackrel{\text { 岁 }}{\stackrel{\text { S }}{=}}$ & 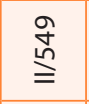 & $\begin{array}{l}\stackrel{g}{\mathrm{H}} \\
\stackrel{\text { I }}{=}\end{array}$ \\
\hline & K\}!|ed!’̣!unس & 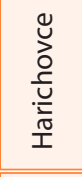 & 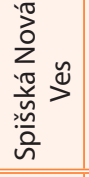 & 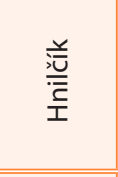 & 总 & 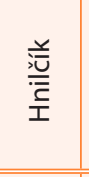 & 总 & $\begin{array}{l}\frac{0}{2} \\
\frac{0}{\frac{0}{0}} \\
\frac{0}{\frac{0}{10}}\end{array}$ & $\begin{array}{l}\frac{0}{0} \\
\frac{0}{\frac{0}{a}} \\
\frac{0}{\frac{10}{2}}\end{array}$ & 离 & $\begin{array}{l}\text { 訔 } \\
\text { है } \\
\text { है }\end{array}$ & $\begin{array}{l}\text { 呞 } \\
\stackrel{\text { है }}{\text { जे }}\end{array}$ & 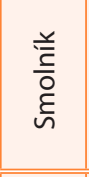 & 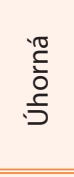 \\
\hline & . ON & - & N & $m$ & + & in & 0 & n & $\infty$ & $a$ & 우 & $=$ & $\simeq$ & $\underline{m}$ \\
\hline
\end{tabular}


It can be assumed that there was a former wooden cross that was replaced by a then popular and long-lasting blackpainted metal-cast cross on a white travertine pedestal. The handcraft detail of the cross is very elaborate and detailed rays symbolise Resurrection; plant motives like vine, clover and laurel symbolise life after death. The writing INRI (lesus Nazarenus Rex ludaeorum) is a biblical reference to Jesus from Nazareththe Nazarene, as King of the Jews. Below the Corpus, there is an inscription medallion (with the date 1907 and a reference to the donors) and a metal-cast relief of Virgin Mary with elaborate plant-motive decoration. The cross is surrounded by a simple metal fence and a group of common lilac (Syringa vulgaris L.).

The oldest preserved crosses documented by Felberová (2010) in Central Spiš date back to the $19^{\text {th }}$ century. These are mainly metal crosses created by the technology of diecasting. They are aesthetically very valuable pieces of art located mainly in cemeteries, but also in open landscapes of Central Spiš.

The roadside Baroque chapel of Saint John of Nepomuk from 1726, located at B-road II/549 (Mníšek nad Hnilcom Krásnohorské Podhradie), at the entrance to villageSmolník (Figure 3) is was registered as a cultural monument since in 1963. It features a typical mining symbol with two hammers at the dating, which represents a symbolic linkage between everyday life and work of locals with their spiritual values. The chapel was built during the reign (1711-1740) of Charles VI (1685-1740) whose name is linked to some of the most spectacular Baroque buildings in the region, in the period when the cult of Saint John of Nepomuk (canonised in 1729) was rising. The development of Smolník continued under the reign (1740-1780) of Maria Theresia (1717-1780) when one of the three mining schools in the Hungarian Kingdom and a mining inspectorate were established.

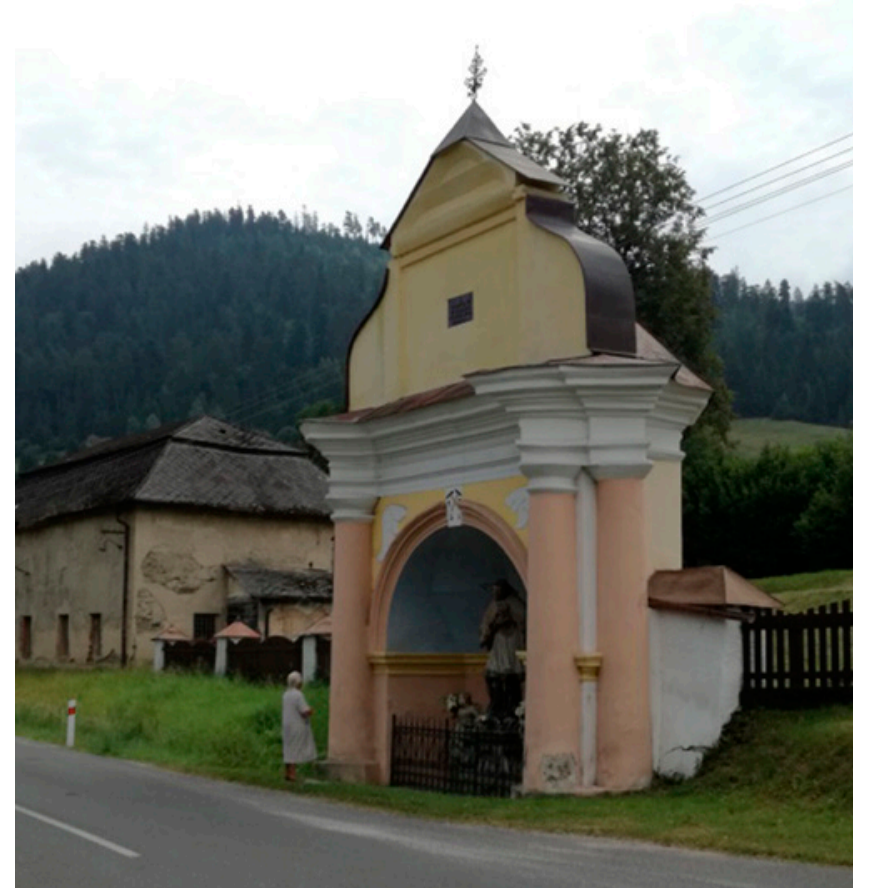

Figure 3 A characteristic Baroque chapel in Smolník (No. 10) with a niche featuring a polychrome statue of Saint John of Nepomuk and decorative mining symbols around the dating Photo: Halajová, 2018
Roadside chapels and shrines often refer to the patrociny patronage of the municipality in which they are located. The patrocinypatronage was usually selected based on local, religious, cultural, economic, and social characteristics. According to Felberová (2010), the patrociny patronage of Saint John of Nepomuk significantly prevails in Central Spiš. This cult was still vivid in the interwar period, when sung litanies where celebrated at small sacral objects of Saint John of Nepomuk in May.

In the studied area, there were woody plants only at roadside crosses - solitary trees at six objects and shrubs or groups of shrubs at three objects (see Table 2). Out of the 14 documented trees, ten 10 individuals are small-leaved lindens (Tilia cordata Mill.), 2 individuals are northern whitecedars (Thuja occidentalis L.), while Norway spruce (Picea abies (L.) H. Karst) and European larch (Larix decidua Mill.) are represented by one individual each. Trees were planted mainly in groups of two, framing the sacral object from both sides (3 samples), there was one object with a group of four trees (two pairs on both sides of the object) and one object with a group of three trees (one tree on both sides of the object and one behind it). The oldest trees with the highest landscape value are small-leaved lindens (Tilia cordata Mill.) - two at the object No. 1 in Harichovce (see figure 4), and two at the object No. 11 in Smolník (see figure 5), all four in the age phase of old/senescent trees that need immediate arborist intervention, in order to be preserved. Shrubs are represented by common lilac (Syringa vulgaris L.), flaky juniper (Juniperus squamata Buch.-Ham. ex D.Don) and box tree (Buxus sempervirens L.). These findings support the results of Kraková (2004), one of the few authors who mapped also vegetation as a part of small sacral monuments and sites, and documented the presence of woody plants at $32 \%$ of small sacral elements in the study area of Central

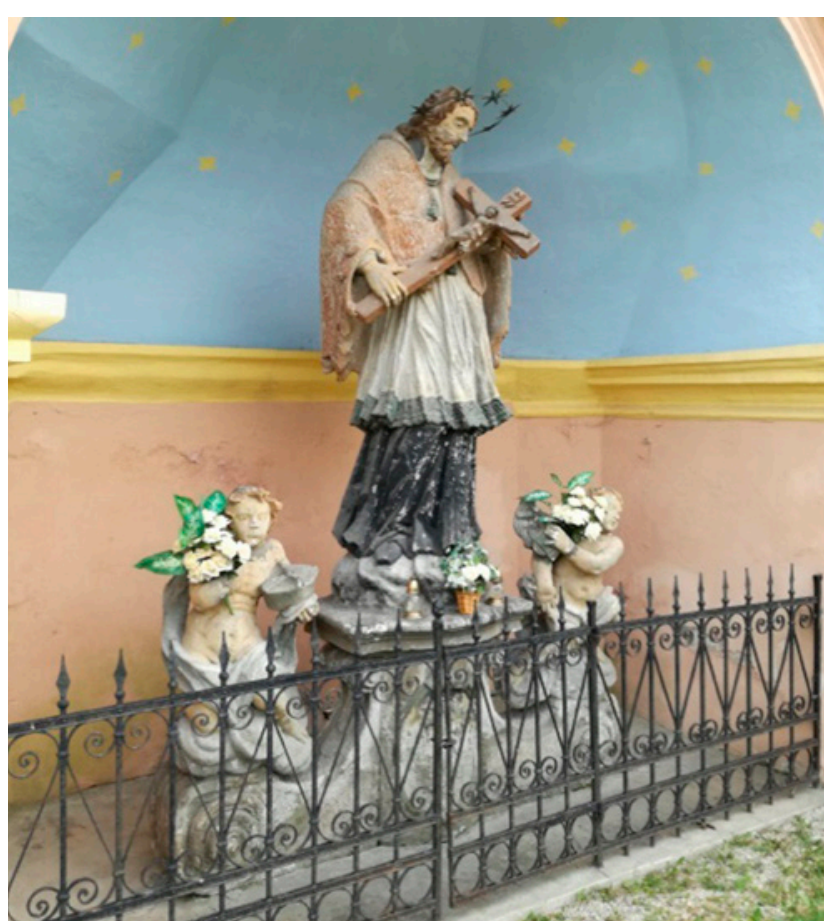

\section{(1)}




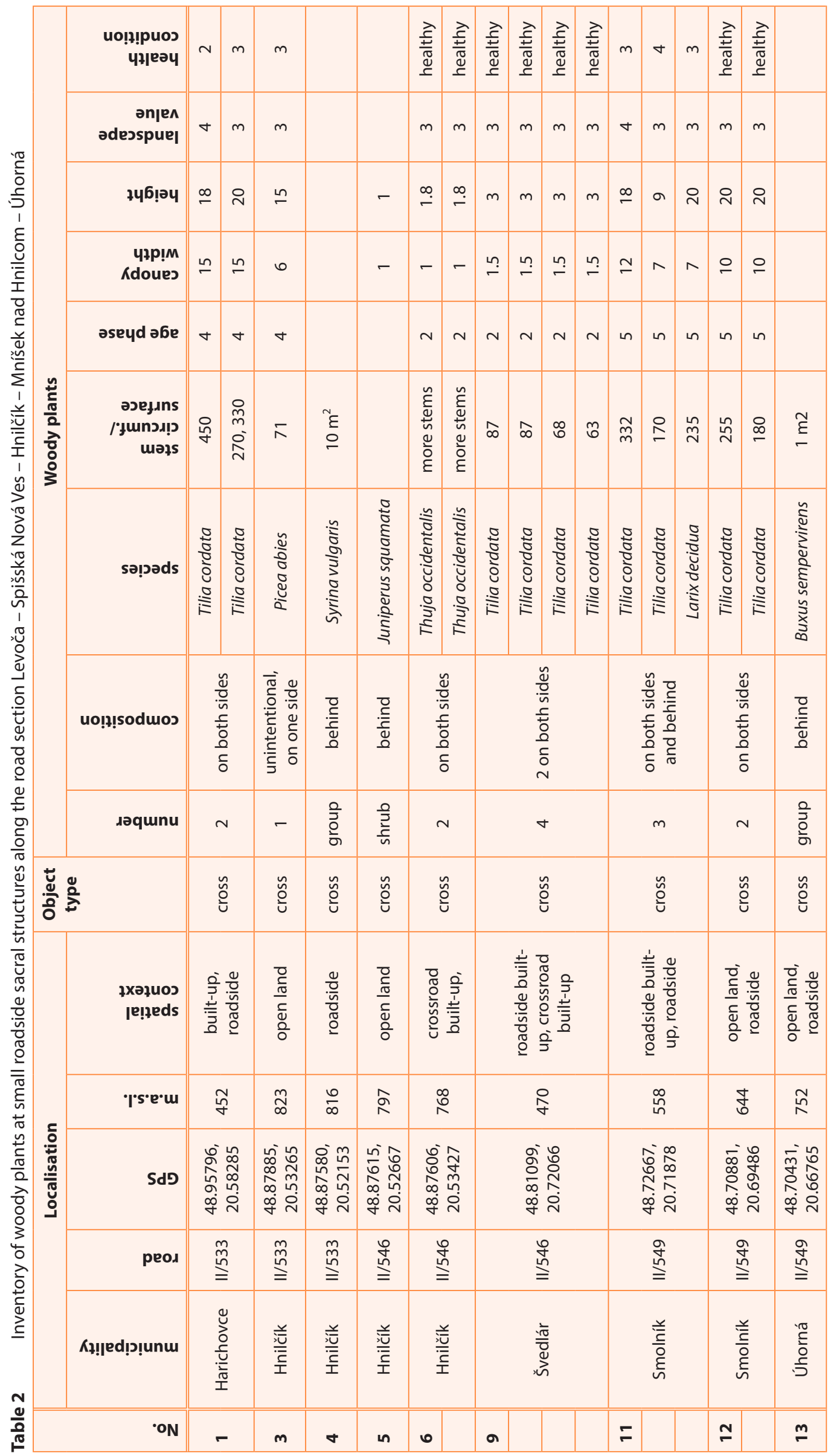




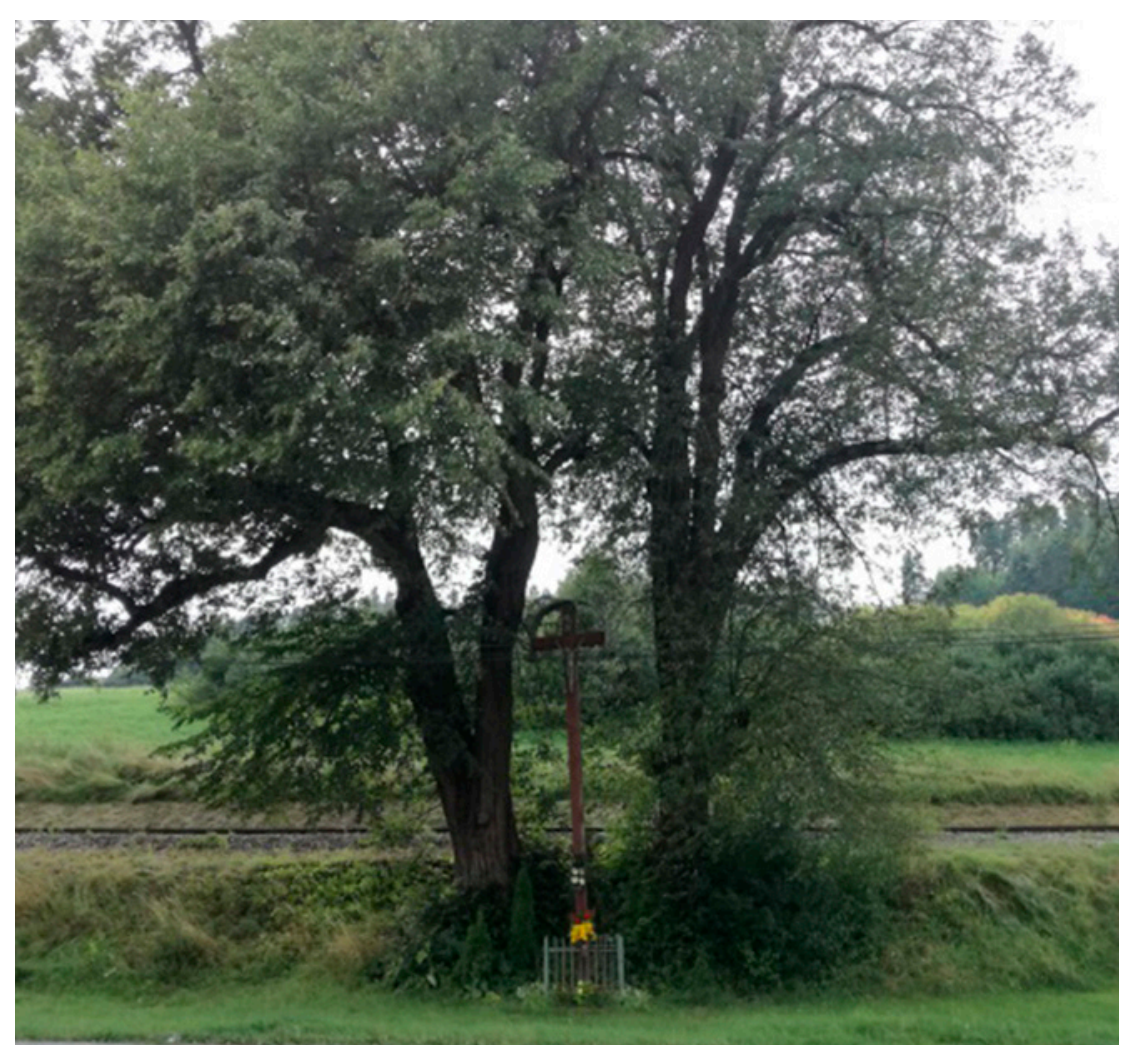

Figure 4 A wooden cross framed by two small-leaved linden trees (Tilia cordata Mill.) in Harichovce (Object No. 1) Photo: Halajová, 2018

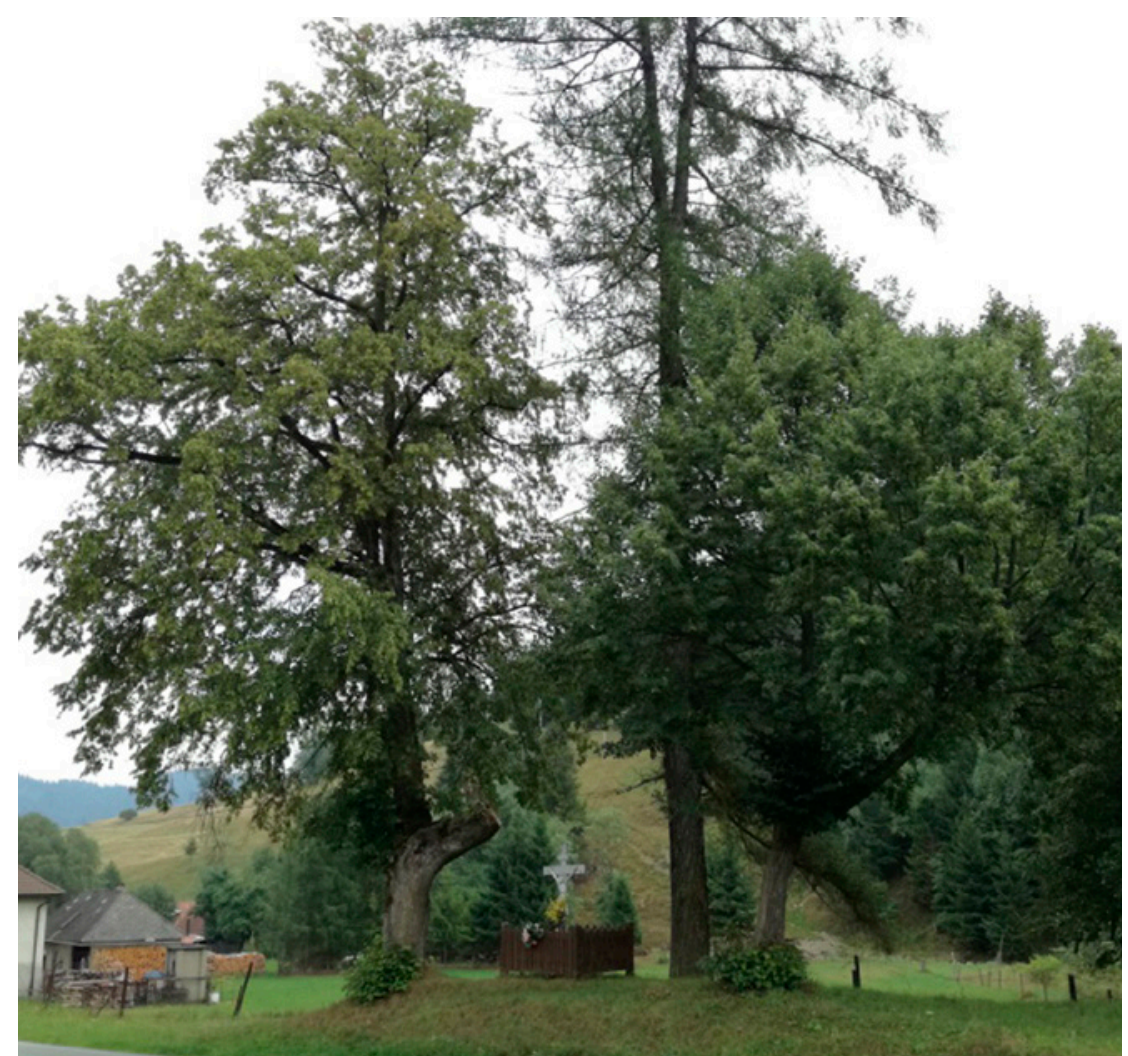

Figure 5 Metal cross in Smolník with two small-leaved linden trees (Tilia cordata Mill.) and a European larch (Larix decidua Mill.) behind the cross (Object No. 11)

Photo: Halajová, 2018
Spiš. In her study, the number of trees per object ranged from one to four, while Tilia (linden) as genus prevailed. Regarding the species composition in our study area, it is important to mention that the planting of nonnative species like Thuja occidentalis L. in open landscapes with important cultural values is to be observed critically, as it changes the traditional character of the landscape.

\section{Conclusion}

Roadside crosses, mainly wooden crosses, are the most common small sacral elements in the observed area of Lower Spiš. Metal crosses on stone pedestals made mainly of travertine show valuable and very elaborate art and handcraft details. Results of the mapping have confirmed that the most common tree genus at objects of small sacral architecture in Spiš is linden (Tilia), while the most represented species is small-leaved linden (Tilia cordata Mill.), mainly in the age phase of mature and old trees. New plantings are rare, while often unsuitable species (e.g. Thuja occidentalis L.) are used. We consider Thuja and other non-native species as unsuitable because they negatively impact the traditional cultural landscape, its visual character and aesthetic values. Their planting shows the lack of professionality and sensitivity in planning and design. When planting trees in rural, especially open landscapes, native tree species should always be preferred. Lower Spiš is widely forested, thus objects are often located on edges of coniferous forests, where sacral elements stand with no additional plantings or they are highlighted with a different species than the prevailing forest species. The most valuable small sacral object is the protected cultural monument of the Baroque roadside chapel of Saint John of Nepomuk from 1726 in Smolník, which reflects the crucial importance of mining tradition in this region during the Baroque period, as well as the popularity of dedicating villages and sacral monuments to this saint. Small sacral objects are part of the mining cultural landscape of Lower Spiš, which are still important elements in the everyday life of local inhabitants. This is reflected by their good technical condition and ornamental decoration with seasonal flowers and candles. 


\section{Acknowledgement}

This paper is an outcome of research projects VEGA 1/0371/18, KEGA 009 SPU-4/2019 and KEGA 011SPU-4/2019.

\section{References}

BIEDERMANN, H. 1992. Lexikón symbolov. Bratislava : Obzor. $373 \mathrm{~s}$. ISBN 80-215-0217-7.

DOHNALOVÁ, B. a i. 2015. Metodika identifikace kulturních artefaktů v krajině. Brno : Mendelova univerzita, 2015, 296 s. ISNB 978-80-7509-382-0.

FELBEROVÁ, M. 2010. Drobná sakrálna architektúra na Strednom Spiši. Levoča : SNM - Spišské múzeum v Levoči, 2010, 14 s. ISBN 978-80-85167-37-5.

HALAJOVÁ, D. - KUBIŠTA, R. 2015. Some Aspects of Greenery Restoration and Maintenance Management of Woody Plants in Cemeteries in Nitra, Slovakia. In Acta Horticulturae et Regiotecturae, vol. 17, 2015, no. 2, pp. 29-34.

JANČURA, P. 2011. Urbanisticko-architektonická štúdia religióznej krajiny Spišský Jeruzalem: krajinárska štúdia. Košický samosprávny kraj, ARLAND s.r.o. Spišská Nová Ves. [online]. [cit. 2019-01-20]. Web: http://www.arland.sk/download/spissky_jeruzalem/sp-jeruzalemsprievodna-sprava.pdf

JANČURA, P. - BOHÁLOVÁ, I. 2001. Identita človeka a krajiny. In Krajina, človek, kultúra, zborník referátov. Banská Bystrica:Slovenská agentúra životného prostredia, 2001, s. 43-55. ISBN80-88850-33-9 JUHÁSOVÁ, G. 2009. Hodnotenie drevín. In Zahradnictví [online], [cit. 2017-02-13]. Available at: http://zahradaweb.cz/ hodnotenie-drevin/

KOPEČEK, P. a i. 2015. Projevy křestananské liturgie v kulturní krajine. Brno : MZLU, 2015. 164 s. ISBN 978-80-7509-387-5.

KRAKOVÁ, A. 2004. Kultúrno-historická krajinná štruktúra stredného Spiša a návrh jej rekonštrukcie: dizertačná práca. Zvolen :TU, 2004 $124 \mathrm{~s}$.

KYSELKA, I. 2014. Drobné historické struktury jako pamět' krajiny a její historická stopa. In Životné prostredie, roč. 48, 2014, č. 1 s. 9-14. ISSN 0044-4863.
MACHOVEC, J. 1987. Hodnocení vzrostlé zeleně v městských pracích. In Životné prostredie, roč. 21, 1987, č. 3, s. 134-139.

MATÁKOVÁ, B. 2011. Drobné sakrálne objekty v krajine Hornej Nitry. Lednice : MZLU, dizertačná práca, 2011, 239 s.

SUPUKA, J.- ŠTEFUNKOVÁ, D. 2014. Kultúrne krajiny a ich historické hodnoty. In Životné prostredie, roč. 48, 2014, č. 1, s. 3-8. ISSN 0044-4863.

ŠIMEK, P. 2001. Hodnocení dřevin a jejich porostů pro pěstební účely v zahradní tvorbě. Lednice : Mendelova univerzita, 2001, $159 \mathrm{~s}$.

TOMAŠKO, I. 2000. Hodnotenie kultúrnej krajiny Spišskopodhradskej kotliny. In Krajina, človek, kultúra, zborník referátov. Banská Bystrica : Slovenská agentúra životného prostredia, 2000, s. 64-68 s. ISBN 80-88850-33-9.

TÓTH, A. 2018. Metodika hodnotenia drobných sakrálnych objektov pre projekt "SakralArch: Zachovanie historického odkazu a architektonickej diverzity drobných sakrálnych stavieb v kultúrnej krajine Slovenska. Rukopis.

TÓTH, A. - FERIANCOVÁ, L. 2016. Restoration of the Landscape Garden in Velká Maňa. In Acta Horticulturae et Regiotecturae, vol. 19, 2016, no. 1, pp. 1-3.

TÓTH, A. - VEREŠOVÁ, M. 2018. Small Sacral Architecture and Trees as Monuments in Diverse Cultural Landscapes of Slovakia. In Plants in Urban Areas and Landscape. Nitra : SUA, 2018, pp. 7-13. ISSN 2585-9811. ISBN 978-80-552-1829-8.

VENCKO, J. 2018. Z dejín okolia Spišského hradu. Bijacovce: Sv. Hubert. n.o. ISBN 978-80-972364-1-0. http://www.obechnilcik. sk/-cirkev

VYHLÁŠKA č. 24/2003 Z.z. Ministerstva životného prostredia Slovenskej republiky, ktorou sa vykonáva zákon č. 543/2002 Z.z. o ochrane prírody a krajiny (v znení neskorších predpisov).

ZÁKON č. 543/2002 Z.z. O ochrane prírody a krajiny (v znení neskorších predpisov). 